\title{
Indirect funduscopy in a gas filled eye: an observation
}

\author{
V T THALLER
}

From Moorfields Eye Hospital, City Road, London EC1V 2PD

SUMMARY It has been observed that a vitrectomised, gas filled, phakic eye can produce a real, inverted, aerial image of the fundus anterior to the cornea. The optics of this phenomenon are discussed.

Indirect funduscopy requires paraxial illumination and a condensing lens positioned at an appropriate distance in front of the eye to produce a real inverted aerial image of the fundus (Fig. 1).

The author has observed that a phakic eye filled with gas after vitrectomy can produce such an indirect' image without the use of a discrete condensing Correspondence to Mr V T Thaller, FRCS.

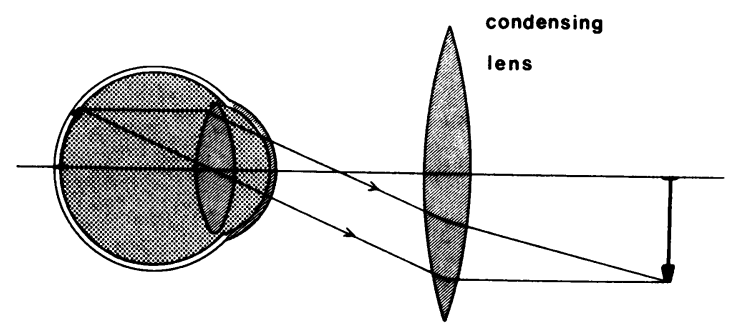

Fig. 1 Image formation by condensing lens in indirect ophthalmoscopy. lens. In this situation the cornea/aqueous/crystalline lens complex together function as a condensing lens (Fig. 2). The image produced has all the properties of an 'indirect' image, being real and inverted, and is formed anteriorly to the cornea. It may be viewed through the operating microscope or slit-lamp microscope by focusing the instrument anteriorly from the cornea on to the image plane. The image may also be photographed by an anterior segment camera (Fig. 3).

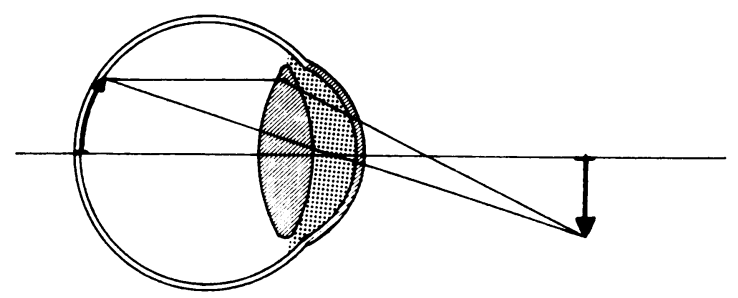

Fig. 2 Image formation by a gas filled phakic eye.

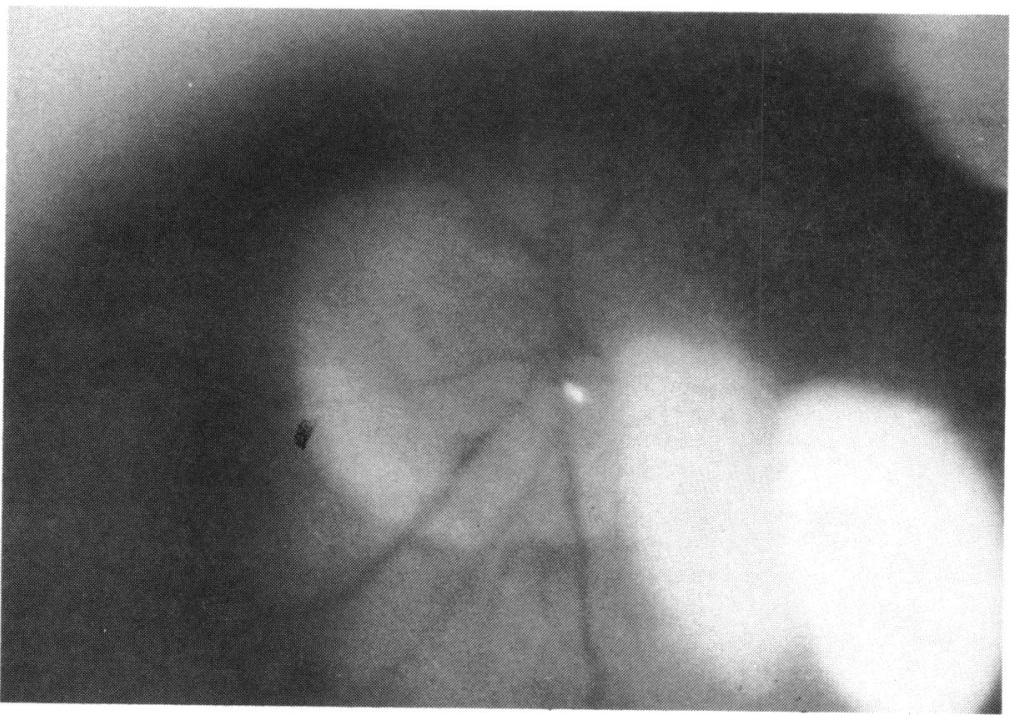

Fig. 3 Slit-lamp photograph of image formed by gas filled phakic eye. Note that plane of focus is anterior to the lids, which appear blurred. The light reflexes are the normal Purkinje images. 


\section{Optics}

The total refractive power of an eye has been calculated by Helmholtz and Gullstrand' to be about +60 dioptres $(+58.64$ dioptres). When the vitreous cavity is filled with air, the posterior lens surface/air interface becomes strongly refractive. Landers et al. ${ }^{2}$ used Gullstrand's model to calculate the refractive power of such a gas filled phakic eye and arrived at a value of approximately +100 dioptres $(+102$ dioptres). The cornea/aqueous/lens complex therefore acts as a +100 -dioptre condensing lens forming an image $\mathrm{I}$ at a distance $\mathrm{V}$ from its nodal point $\mathrm{N}_{1}$ (Fig. 4). The position of this image is related to the distance $U$ between the retina $(\mathrm{O})$ and nodal point $\mathrm{N}_{2}$ by equation (1):

$$
D=1 / v+1 / v
$$

where $\mathrm{D}$ is the dioptric power of the refracting complex. From equation (1) it can be established that for the image to be formed at infinity $(1 / v=0)$ the object distance must be $10 \mathrm{~mm}$ :

$$
D=1 / 2 \therefore U=1 / 0=1 / 100=0.01 \mathrm{~m} \text {. }
$$

The value of $U$ in the emmetropic eye is nearer $20 \mathrm{~mm}$. In this instance the image is formed at $20 \mathrm{~mm}$ :

$$
\mathrm{V}=\frac{1}{(\mathrm{D}-1 / \mathrm{v})}=\frac{1}{100-50}=0.02 \mathrm{~m} \text {. }
$$

It follows that in myopic eyes which have a higher

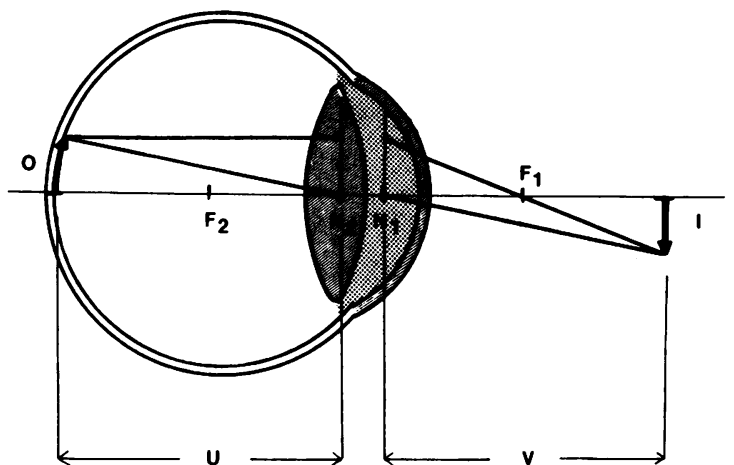

Fig. 4 The optics of fundus image projection by a gas filled phakic eye.

value of $U$ the image will be correspondingly closer to $F_{1}$.

The clinical usefulness of this phenomenon of 'auto indirect ophthalmoscopy' of a gas filled eye remains to be established.

\section{References}

1 Helmholtz H. Treatise on physiological optics. New York: Dover, 1924: 1.

2 Landers MB, Stefansson E, Wolbarsht ML. The optics of vitreous surgery. Am J Ophthalmol 1981; 91: 611-4.

Accepted for publication 27 June 1985. 